\title{
The range of a planar function with ambiguous points
}

\author{
by \\ J. Gresser (Milwaukee, Wis.)
}

By an are $\Lambda$ at a point $\zeta$ in the plane $P$ we shall mean a simple continuous curve $z=z(t)(0 \leqslant t<1)$ such that $z(t) \neq \zeta$ for $0 \leqslant t<1$ and $\lim z(t)=\zeta$. Let $w=f(z)$ be an arbitrary single valued function mapping $P$

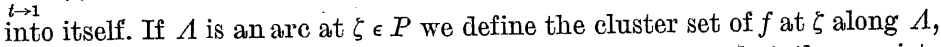
denoted by $C_{A}(f, \zeta)$, to be the set of all values $a$ such that there exists a sequence $\left\{z_{n}\right\}$ on $\Lambda$ with $z_{n} \rightarrow \zeta$ and $f\left(z_{n}\right) \rightarrow a$. We call $\zeta$ an ambiguous point of $f$ if there exist ares $\Lambda$ and $\Lambda^{\prime}$ at $\zeta$ such that $C_{\Lambda}(f, \zeta) \cap C_{A^{\prime}}(f, \zeta)$ $=\emptyset$. If in addition $\Lambda$ and $\Lambda^{\prime}$ are rectilinear segments having opposite directions then $\zeta$ is called a rectilinearly oppositely ambiguous point, and the angle $\theta$ between $\Lambda$ or $\Lambda^{\prime}$ and a horizontal line shall be called the corresponding direction of ambiguity. We shall let $f(r, s)$ denote the following sentence: Given the distinct directions $\theta_{1}, \theta_{2}, \ldots, \theta_{r}$, there exists a single valued function $f$ having a range of at most $s$ values, such that every point in the plane is a rectilinearly oppositely ambiguous point of $f$ with at least one of $\theta_{1}, \theta_{2}, \ldots, \theta_{r}$ as direction of ambiguity.

The intent of this paper is to answer a question which arises from the following two theorems.

THEOREMI 1. If $2^{*_{0}} \leqslant s_{1}$ then $f(3,4)[1]$.

THEOREM 2. If $2^{\mathrm{K}_{0}} \leqslant \kappa_{n}$ then $f\left(n+2,2^{n+2}-1\right)$ [2].

It is evident that for $n=1$ Theorem 2 is a weaker form of Theorem 1 . We intend to show that Theorem 2 remains true for a range of $2^{n+1}$ values.

Let $X_{n}$ be the set consisting of all $n+2$ tuples of the form $\left(j_{1}, j_{2}, \ldots\right.$ $\left.\ldots, j_{k-1}, k, j_{k+1}, \ldots, j_{n+2}\right)$ where $k=1,2,3, \ldots, n+2$ and each $j_{i}$ is zero or one. For any $n+2$ tuple of the form $\left(j_{1}, j_{2}, \ldots, j_{n+2}\right)$ where each $j_{i}$ is zero or one we define the subset $B\left(j_{1}, j_{2}, \ldots, j_{n+2}\right)$ of $X_{n}$ to consist of the elements

$$
\begin{aligned}
& \left(1, j_{2}, j_{3}, \ldots, \quad j_{n+2}\right), \\
& \left(j_{1}, 2, j_{3}, \ldots, \quad j_{n+2}\right), \\
& \left(j_{1}, j_{2}, j_{3}, \ldots, j_{k-1}, k, j_{k+1}, \ldots, j_{n+2}\right), \\
& \left(j_{1}, j_{2}, j_{3}, \ldots, \quad j_{n+1}, n+2\right) .
\end{aligned}
$$


Let $B_{1}=B\left(j_{1}, j_{2}, \ldots, j_{n+2}\right)$ and $B_{2}=B\left(j_{1}^{\prime}, j_{2}^{\prime}, \ldots, j_{n+2}^{\prime}\right)$. If $j_{i} \neq j_{i}^{\prime}$ for at least two values of $i$ then $B_{1} \cap B_{2}=\varnothing$.

LemMa. Let $Y_{n}$ be the set of all $n+2$ tuples having coordinates that are zero or one. For any natural number $n$, there exists a subset $A_{n}$ of $Y_{n}$ containing exactly $2^{n+1}$ elements, such that any two elements of $A_{n}$ differ in at least two coordinates.

We shall actually prove the existence of two such disjoint sets, both satisfying the conditions of the lemma, and our proof is by induction.

For $n=1$ let $A_{1}=\{(0,0,0),(0,1,1),(1,1,0),(1,0,1)\}$ and $B_{1}=\{(1,1,1),(1,0,0),(0,0,1),(0,1,0)\}$. Then $A_{1} \cap B_{1}=\varnothing$ and $A_{1}$ and $B_{1}$ both satisfy the conditions of the lemma.

Let $n \geqslant 1$ and suppose $A_{n}$ and $B_{n}$ are disjoint sets, both satisfying the conditions of the lemma. Let

$$
\begin{aligned}
& C=\left\{\left(j_{1}, j_{2}, \ldots, j_{n+2}, 0\right) \mid\left(j_{1}, j_{2}, \ldots, j_{n+2}\right) \in A_{n}\right\}, \\
& D=\left\{\left(j_{1}, j_{2}, \ldots, j_{n+2}, 1\right) \mid\left(j_{1}, j_{2}, \ldots, j_{n+2}\right) \in B_{n}\right\}, \\
& D=\left\{\left(j_{1}, j_{2}, \ldots, j_{n+2}, 0\right) \mid\left(j_{1}, j_{2}, \ldots, j_{n+2}\right) \in B_{n}\right\}, \\
& F=\left\{\left(j_{1}, j_{2}, \ldots, j_{n+2}, 1\right) \mid\left(j_{1}, j_{2}, \ldots, j_{n+2}\right) \in A_{n}\right\}
\end{aligned}
$$

and set $A_{n+1}=C \cup D$ and $B_{n+1}=E \cup F$. Then $A_{n+1} \cap B_{n+1}=\emptyset$ and both satisfy the conditions of the lemma, which completes the proof.

Now associated with the $2^{n+1}$ elements of $A_{n}$ there are $2^{n+1}$ sets of the form $B\left(j_{1}, j_{2}, \ldots, j_{n+2}\right)$ where $\left(j_{1}, j_{2}, \ldots, j_{n+2}\right) \in A_{n}$ which for convenience we label arbitrarily as $B_{1}, B_{2}, \ldots, B_{2^{n+1}}$. By the above comment the $B_{i}$ 's are mutually disjoint since elements of $A_{n}$ differ in at least two coordinates, and so $\bigcup_{i=1}^{2 n+1} B_{i}$ has $(n+2) 2^{n+1}$ elements. Since $X_{n}$ has $(n+2) 2^{n+1}$ elements, it follows that $X_{n}=\bigcup_{i=1}^{2^{n+1}} B_{i}$.

THEOREM 3. If $2^{\aleph_{0}} \leqslant \aleph_{n}$, then $f\left(n+2,2^{n+1}\right)$.

Proof. With the above work behind us our proof essentially parallels the proof of Theorem 1. By a theorem of Davies [3] the hypothesis implies that $P=\bigcup_{j=1}^{n+2} E_{j}$ where the $E_{j}$ 's are mutually disjoint and every line having direction $\theta_{j}$ intersects $E_{j}$ in at most finitely many points. Let $L$ be any line in the plane. We impose an ordering on $L$ as follows. For $z, z^{\prime} \in L$ $z<z^{\prime}$ if either $\operatorname{Im}(z)<\operatorname{Im}\left(z^{\prime}\right)$ or if $\operatorname{Im}(z)=\operatorname{Im}\left(z^{\prime}\right)$ and $\operatorname{Re}(z)<\operatorname{Re}\left(z^{\prime}\right)$. Let $L\left(\theta_{j}\right)$ be any line in the plane having direction $\theta_{j}$. Then $L\left(\theta_{j}\right)$ intersects $E_{j}$ in only finitely many points which we label as $e_{1}<e_{2}<e_{3}<\ldots$ $<e_{m}$. Let $z \in L\left(\theta_{j}\right)$. We call $z$ a class zero point with respect to $E_{j}$ if either $z<e_{1}, e_{2}<z<e_{3}, e_{4}<z<e_{5}, \ldots, e_{p}<z<e_{p+1}(p \leqslant m$ and $p$ even $)$ and we call $z$ a class one point with respect to $E_{j}$ if either $e_{1}<z<e_{2}, e_{3}<z<e_{4}$, $e_{5}<z<e_{6}, \ldots, e_{p}<z<e_{p+1}(p \leqslant m$ and $p$ odd $)$. For $p=m$ we define $e_{p}<z<e_{p+1}$ to mean $e_{p}<z$. If $L\left(\theta_{j}\right) \cap E_{j}$ is empty then we call $z \in L\left(\theta_{j}\right)$ a class zero point with respect to $E_{j}$. We have thus partitioned every line in the plane having direction $\theta_{1}, \theta_{2}, \ldots, \theta_{n+2}$ into alternating intervals of class zero or class one points. Now let $z \in P$. Then $z \in E_{k}$ for some unique $k$. Through $z$ construct the $(n+1)$ lines having directions $\theta_{j}$ with $j \neq k$, say $L\left(\theta_{j}\right)(j \neq k)$. For each $j \neq k$ since $z \notin E_{j}, z$ is a class zero or a class one point with respect to $\mathbb{E}_{j}$. We define the correspondence $\varphi$ mapping the plane into $X_{n}$ by $\varphi(z)=\left(j_{1}, j_{2}, \ldots, j_{k-1}, k, j_{k+1}, \ldots, j_{n+2}\right)$ where $z \in \mathbb{E}_{k}$ and $z$ is a class $j_{i}$ point with respect to $E_{i}(i \neq k)$.

Using the $B_{j}$ 's defined previously and the correspondence $q$ we are now ready to define our function $f$. For $z \in P$ define $f(z)=j$ where $\varphi(z) \in B_{j}$. The function $f$ is clearly single valued and has a range of at most $2^{n+1}$ values.

Let $z \in P$. Then $z \in E_{k}$ for some unique $k$. Let $L\left(\theta_{k}\right)$ be a line segment with direction $\theta_{k}$ containing $z$ in its interior and small enough so that it intersects $E_{k}$ in no other point. Let $z_{1}, z_{2} \in L\left(\theta_{k}\right)$ with $z_{1}<z<z_{2}$. Then $\varphi\left(z_{1}\right)$ and $\varphi\left(z_{2}\right)$ differ in their $k$ th coordinates. We assert that $f\left(z_{1}\right) \neq f\left(z_{2}\right)$, for if not $f\left(z_{1}\right)=f\left(z_{2}\right)=j$ implies that $\varphi\left(z_{1}\right), \varphi\left(z_{2}\right) \in B_{j}$ and since $\varphi\left(z_{1}\right)$ and $\varphi\left(z_{2}\right)$ differ in their $k$ th coordinates it follows from the definition of $B_{j}$ and $\varphi$ that one of the elements $z_{1}$ or $z_{2}$ must be in $E_{k}$, which contradicts the definition of $L\left(\theta_{k}\right)$. Thus if we let $\Lambda$ and $\Lambda^{\prime}$ be the ares at $z$ determined by the two sides of $L\left(\theta_{k}\right)$ we see that $C_{A}(f, z) \cap C_{A^{\prime}}(f, z)=\emptyset$ and hence $z$ is a rectilinearly oppositely ambiguous point of $f$ with $\theta_{k}$ as direction of ambiguity.

It remains an open question whether the range can be reduced further. The function we have defined in Theorem 3 depends upon the partition of $X_{n}$ into the $2^{n+1}$ disjoint sets $B_{j}$ and the question immediately arises as to whether or not we can find a partition of $X_{n}$ into say $m$ disjoint sets, with $m<2^{n+1}$, that would yield a function satisfying Theorem 3 but with a range of at most $m$ values. It can be shown however, that such an accomplishment would require additional, as yet unknown, information about Davies' decomposition of the plane mentioned at the beginning of the proof of Theorem 3 .

There is in fact reason to suspect that the range cannot be further reduced. We define the minimal range as the smallest number $\varrho_{n}$ such that $2^{\mathrm{x}_{0}}=\mathrm{s}_{n}$ implies that $f\left(n+2, \varrho_{n}\right)$. It has been shown in [1] that there does not exist a function having a range of three values or less such that every point in the plane is a rectilinearly oppositely ambiguous point. Note that we make no restriction here on directions of ambiguity. Thus $\varrho_{1}=4$ and so Theorem 3 is minimal for $n=1$.

The reader is referred to [1] and [2] for other interesting theorems on the subject. In particular $f\left(n+2, s_{0}\right)$ implies that $2^{x_{0}} \leqslant s_{n}$, so that the converses to all the above results hold. 


\section{References}

[1] F. Bagemihl and S. Koo, The continuum hypothesis and ambiguous points of planar functions, Zeitschr. f. math. Logik und Grundlagen d. Math. 13 (1967) pp. 219-223.

[2] F. Bagemihl, The hypothesis $2^{\mathrm{K}_{0}} \leqslant \mathrm{~N}_{n}$ and ambiguous points of planar functions, Fund. Math. 61 (1967) pp. 73-77.

[3] R. O. Davies, The power of the continum and some proposition of plane geometry, Fund. Math. 52 (1963), pp. 277-281.

\section{On countable multiple point compactifications*}

by

\section{A. K. Steiner and E. F. Steiner (Ames, Iowa)}

Throughout this paper a space $X$ always denotes a Tychonoff space and $\hat{X}$ a Hausdorff compactification of it. The Stone-Čech compactification will be denoted by $\beta X$. Countable will mean countably infinite.

In [4], Magill characterizes those spaces $X$ which possess finite compactifications (i.e. $|\hat{X}-X|$ is finite) and in [5], those which possess countable compactifications (i.e. $|\hat{X}-X|$ is countable). A much wider class of compactifications consists of those having a finite or countable number of multiple points as defined by Njåstad, [6].

In Section 1 of this paper we show that every non pseudocompact space has countable multiple point compactifications. It then follows that although the Euclidean $n$-spaces do not admit countable compactifications (cf. [5]) they do have countable multiple point compactifications. Section 1 will also provide examples of spaces $X$ such that $|\beta X-X|$ is infinite yet $X$ does not possess a countable multiple point compactification. These examples are obtained by using the fact that for any space $Y$, there exists a space $X$ such that $\beta X-X=Y$ (cf. [2], p. 133).

Recent work (cf. [1], [3], [6], [7], [8]) has shown that many compactifications are of the Wallman-type (henceforth called Wallman compactifications) as defined by Frink [1]. In [6], Njåstad shows that if the set of multiple points in $\hat{X}$ is contained in some subset of $\hat{X}-X$ which is normally and zero-dimensionally embedded in $\hat{X}$, then $\hat{X}$ is a Wallman compactification. It follows that all finite multiple point compactifications are Wallman. The authors, in a previous work [8], have shown that every countable compactification is Wallman. The purpose of Section 2 is to generalize this by showing that all countable multiple point compactifications are Wallman. Since there are examples where the countable set of multiple points is not contained in any subset of $\hat{X}-X$ which is normally and zero-dimensionally embedded in $\hat{X}$, this does not follow from the theorem of Njaistad.

* Research supported by the National Science Foundation, Grant GP 6529. 